\title{
Generación Eléctrica a Partir de la Fotosíntesis Natural; ¿Una Realidad Escalable?
}

\section{Electricity Generation from Natural Photosynthesis; A Scalable Reality?}

\author{
ZAPIEN-RODRÍGUEZ, José Manuel ${ }^{*}$, SOLORIO-DE JESÚS, Bianca Azucena, BALLESTEROS- \\ PACHECO, Juan Carlos y NÚÑEZ-AYALA, Frida Libertad
}

Universidad Politécnica de Lázaro Cárdenas, Ingeniería Mecatrónica - Ingeniería en Tecnología Ambiental. Av. Galeanas Sin Número, Colonia Las 600 Casas, Cd. Lázaro Cárdenas, Michoacán. C.P. 60950

ID $1^{\mathrm{er}}$ Autor: José Manuel, Zapien-Rodríguez, / ORC ID: 0000-0001-7198-2118, CVU CONACYT ID: 228342

ID $1^{\text {er }}$ Coautor: Bianca Azucena, Solorio-De Jesús / ORC ID: 0000-0001-9669-0410

ID $2^{\text {do }}$ Coautor: Juan Carlos, Ballesteros-Pacheco / ORC ID: 0000-0001-9684-6438, CVU CONACYT ID: 57746

ID $3^{\text {er }}$ Coautor: Frida Libertad, Núñez-Ayala / ORC ID: 0000-0003-1933-2964

DOI: $10.35429 / J R E .2019 .10 .3 .1 .6$

Recibido 16 de Junio, 2019; Aceptado 30 Septiembre, 2019

\begin{abstract}
Resumen
A nivel mundial hay 7,5 mil millones de habitantes, y el $16 \%$ de esta población carece del servicio de electricidad, es por ello que utilizan combustibles fósiles, para calentar y alumbrar sus hogares. El elevado consumo de energía mundial propicia que la contaminación en general de la tierra y atmosfera sea elevada, con graves resultados para los ecosistemas, la salud de las personas y el clima, debido a que las principales fuentes de energías empleadas son No Renovables. La energía procedente del sol la usan directamente las plantas verdes y demás organismos fotosintéticos capaces de capturar la energía solar, transformarla en energía química y almacenarla. Este proceso natural se denomina fotosíntesis y consiste en convertir la energía solar, el agua y el dióxido de carbono en carbohidratos y oxígeno que actúan como nutrientes para las plantas. La tecnología del proceso de la fotosíntesis está basada en un generador biológico que obtiene electricidad de la descomposición de sustancias orgánicas extraídas naturalmente de las plantas. Lo consigue liberando electrones y $\mathrm{H} 2 \mathrm{O}$ en el proceso, sin causar daños a ningún organismo vivo. Este proyecto permite identificar nuevas formas de generación de energía limpia, así como la investigación de energías alternativas sustentables.
\end{abstract}

Generación Eléctrica, Fotosíntesis, Energías Renovables

\begin{abstract}
Worldwide there are 7.5 billion population, and $16 \%$ of this people does not have electricity service, therfore they use fossil fuels to heat and light their homes. The high energy consumption at world, causes a high pollution in general of the earth and atmosphere, with serious results for the ecosystems, the health of the people and the climate, due to the main sources of energy used are NonRenewable. The solar energy is directly used by green plants and other photosynthetic organisms, capable of capturing solar energy, transforming it into chemical energy and storing it. This natural process is called photosynthesis and consists of converting solar energy, water and carbon dioxide into carbohydrates and oxygen that act as nutrients for plants. The photosynthesis process technology is based on a biological generator that obtains electricity from the decomposition of organic substances extracted naturally from plants. It do it by releasing electrons and $\mathrm{H} 2 \mathrm{O}$ in the process, without causing damage to any living organism. This project allows to identify new ways to generate clean energies, as well as the investigation of sustainable alternative energies.
\end{abstract}

Electric Generation, Photosynthesis, Renewable Energies

Citación: ZAPIEN-RODRÍGUEZ, José Manuel, SOLORIO-DE JESÚS, Bianca Azucena, BALLESTEROS-PACHECO, Juan Carlos y NÚÑEZ-AYALA, Frida Libertad. Generación Eléctrica a Partir de la Fotosíntesis Natural; ¿Una Realidad Escalable? Revista de Energías Renovables 2019. 3-10: 1-6

*Correspondencia al Autor (Correo electrónico: zapien_jomazaro@uplc.edu.mx)

$\dagger$ Investigador contribuyendo como primer autor. 


\section{Introducción}

La energía eléctrica es una forma de manifestación de la energía natural y es de gran importancia para el ser humano, sin embargo el sector industrial es quien requiere una gran cantidad en comparación con el consumo residencial promedio.

Los recursos energéticos fósiles, como su nombre lo indica, tienen una cantidad límite para ser empleado por la humanidad, ya que son de carácter no renovable. Se estima que dentro de 50 años la extracción de petróleo se encuentre comprometida, debido no solo a su uso en el área eléctrica, si por el uso desmedido de plásticos desechables.

El elevado consumo de hidrocarburos a nivel mundial, propicia la contaminación tanto del aire, tierra y agua a niveles muy elevados, con graves resultados para los ecosistemas y seres vivos que habitamos en el planeta.

El aumento de la demanda eléctrica conlleva a la continua investigan en energías alternativas, enfocándose en las renovables, con la finalidad de suministrar los requerimientos eléctricos en un sistema de generación distribuida a baja potencia de acuerdo a las condiciones particulares de cada consumidor.

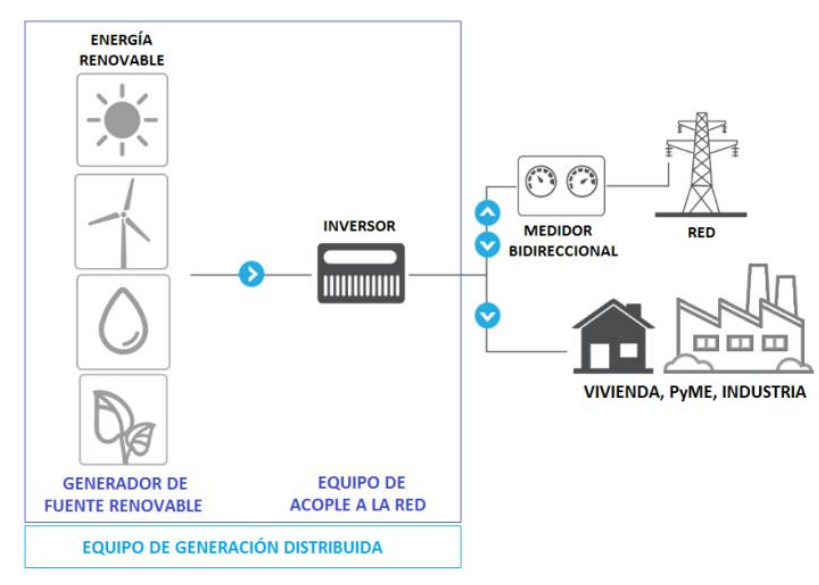

Figura 1 Generación Distribuida

Fuente: https://energialimpiaparatodos.com

Los continuos avances en la investigación aplicada de energías renovables tales como Sistemas Fotovoltaicos y Parques Eólicos, abrieron la puerta al uso de recursos naturales para la adquisición de energía eléctrica, un primer recurso empleado fue el uso de los residuos orgánicos para aprovechar el poder calorífico de estos al momento de hacer combustión.
Generar electricidad a partir de un recurso tan necesario para la humanidad como lo es la vegetación, sin comprometer la estructura orgánica de la naturaleza, sería un parteaguas en el desarrollo evolutivo de la especie, esta idea surgió hace no más de una década, dando como resultado un futuro promisorio, donde hasta el momento se han conectado cargas que no requieren más de 5 volts, sin embargo la tecnología aún está lejos de ser considerada para cargas que requieren de mayor potencia eléctrica.

Asegurar los requerimientos eléctricos no solo es cuestión de generar electricidad por medio de las diferentes tecnologías disponibles, se debe además eficientar los sistemas para evitar las pérdidas eléctricas y aprovechar al máximo los recursos disponibles.

El desarrollo de este proyecto busca que la concientización de las personas hacia nuevas fuentes alternativas de energía limpia y se opte por hacer uso de ellas, evidenciando no solo los beneficios económicos que conlleva al reducir el consumo eléctrico convencional, además de la contribución ambiental mediante el cuidado de áreas verdes que son sinónimo de vida por la producción de oxígeno.

Las plantas por medio de sus hojas captan la energía proveniente del sol (energía lumínica) y la trasforman en energía química para posteriormente generar una corriente eléctrica por medio del proceso de la fotosíntesis. Para captar los electrones que libera la planta al hacer su rizo deposición, se implementa una pila eléctrica, que transforma la energía química en energía eléctrica.

Durante el proceso natural de la fotosíntesis, se genera la materia orgánica que requieren las plantas para su crecimiento normal, sin embargo el excedente de alimento se distribuye a lo largo de sus raíces donde los microorganismos lo aprovechan.

El procesos de la fotosíntesis se lleva a cabo por medio de la excitación de electrones, por lo que captar estos electrones excedentes al hacer la rizo deposición, se implementa una pila eléctrica por medio de un par de electrodos, que permite transformar la energía química en energía eléctrica. 


\section{Fotosíntesis}

La fotosíntesis es un proceso metabólico que llevan a cabo algunas células de organismos autótrofos para sintetizar sustancias orgánicas a partir de otras inorgánicas. Este proceso convierte la energía luminosa captada por las hojas en energía química estable.

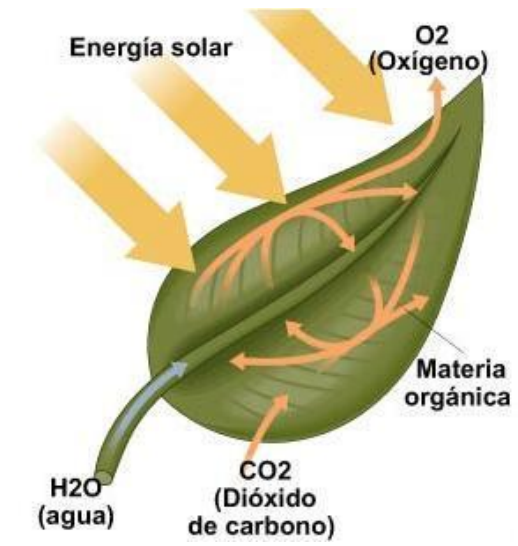

Figura 2 La Fotosíntesis como Proceso Metabólico Fuente: https://sites.google.com

La fotosíntesis se lleva a cabo mediante dos etapas: La lumínica, en la que se utiliza la energía de la luz para sintetizar el ATP y NADPH, y la etapa Fijadora de Carbono, en donde se realiza la producción de azucares necesarios para el crecimiento de la planta.

Los organismos fotosintéticos capturan la luz a través de una red de pigmentos que se ubican en los tilacoides del cloroplasto formando complejos recolectores de luz, los cuales fluyen por una pendiente energética hacia un par especial de moléculas de clorofilas a colectoras de energía que junto a proteínas asociadas constituyen el centro de reacción.

Cuando la clorofila o el centro de reacción reciben la energía de las moléculas excita a uno de sus electrones y sale saltando al sistema de transporte de electrones que se encuentra próximo a ella y así se inicia un flujo de electrones.

En el proceso de fotosíntesis, las plantas expulsan una serie de residuos en forma de moléculas de metabolitos, posteriormente una serie de bacterias o microorganismos rompen estas moléculas para liberar electrones, que serán captados a través de los electrodos para la generación eléctrica.
El dióxido de carbono se fija y se libera como exudados de las raíces por las plantas y es utilizado por microorganismos que devuelven el dióxido de carbono a la atmósfera. Los microorganismos usan el ánodo como aceptor de electrones para obtener energía metabólica. Estos electrones fluyen debido a la diferencia de potencial, desde el ánodo a través de un circuito eléctrico con una carga o una resistencia al cátodo, por lo tanto se genera electricidad que se puede usar

El tamaño de la planta y su capacidad de generar metabolitos incide directamente en la cantidad de electrones desprendidos, de modo que teóricamente es un método escalable, que puede emplearse tanto en una pequeña maceta como en el techo de una casa, incluso un jardín urbano o un parque de mayor dimensiones.

\section{Fundamentos de la experimentación}

El proceso de genera electricidad de las plantas, no afecta en su desarrollo natural ni al ambiente donde se realizó la experimentación. La corriente eléctrica depende de la eficiencia fotosintética de las plantas, la rizo deposición así como de la eficiencia energética de los microorganismos de la misma.

El desarrollo del proyecto se basa en la reacción de la fotosíntesis la cual se describe como:

$6 \mathrm{CO}_{2}+12 \mathrm{H}_{2} \mathrm{O} \rightarrow \mathrm{C}_{6} \mathrm{H}_{12} \mathrm{O}_{6}+6 \mathrm{O}_{2}+6 \mathrm{H}_{2}$

Las plantas empleadas en la experimentación son las de mayor predominio en la región, debido a la capacidad de adaptarse a climas cálidos.

Los electrodos empleados son el cobre como ánodo y el zinc como cátodo, debido a que su composición electroquímica permite una reacción redox, donde la oxidación (perdida de electrones) y la reducción (ganancia de electrones) se realiza debido a la diferencia de potencial eléctrico entre los electrodos.

La reacción electroquímica en los electrodos se muestra a continuación:

Ánodo

$2 \mathrm{C}_{6} \mathrm{H}_{12} \mathrm{O}_{6} \rightarrow 2 \mathrm{C}_{6} \mathrm{H}_{10}+4 \mathrm{H}^{+}+4 e^{-}$ 


\section{Cátodo}

$\mathrm{O}_{2}+4 \mathrm{H}^{+}+4 e^{-} \rightarrow 2 \mathrm{H}_{2} \mathrm{O}$

Reacción Continua de la Red

$$
2 \mathrm{C}_{6} \mathrm{H}_{12} \mathrm{O}_{6}+\mathrm{O}_{2} \rightarrow 2 \mathrm{C}_{6} \mathrm{H}_{10} \mathrm{O}_{6}+2 \mathrm{H}_{2} \mathrm{O}
$$

Cuando los ánodos y cátodos se colocan cerca de las raíces de la planta, los electrones son atraídos a través del ánodo debido a la carga positiva y los protones son atraídos a través del cátodo debido a su carga negativa.

\section{Metodología}

Para la realización del proyecto se hicieron pruebas eléctricas con el fin de que corroborar las reacciones electroquímicas que generen el flujo de electrones esperado por la fotosíntesis natural de las plantas a través de los electrodos.

En una primer instancia se replicó el proyecto en una planta de fácil acceso y dimensiones pequeñas para todas las casas habitación, debido a que no requiere de una gran cantidad de cuidados, además que es muy común encontrarlas por su tipo de flor que es atractiva para la decoración del hogar, esta es la Margarita (Bellis Perennis).

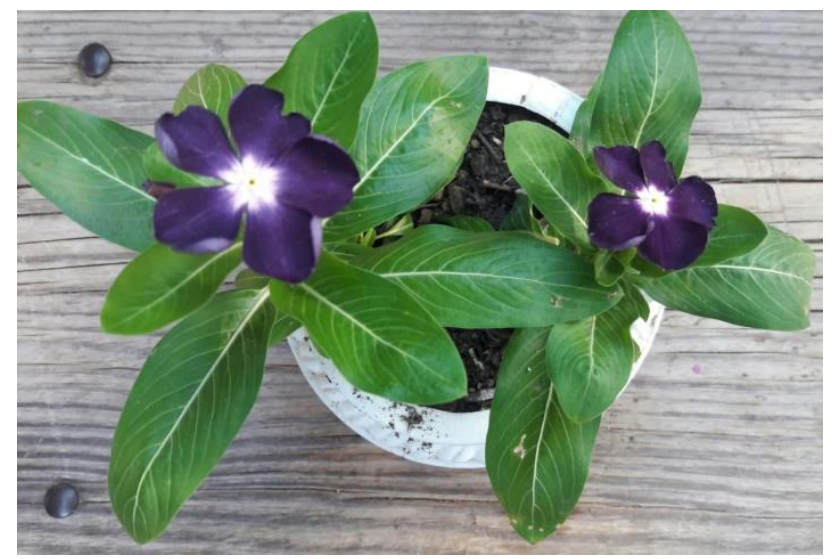

Figura 3 Planta de prueba (Margarita - Bellis Perennis) Fuente: Elaboración Propia

La planta de las margaritas cuenta con un tamaño idóneo que permite un crecimiento en espacios reducidos (macetas), lo que permite que sus raíces abarquen todo el espacio en el que se encuentren, de esta manera los electrodos están en mayor contacto con los microorganismos que realizan la liberación de electrones.
Las conexiones internas que se realizaron para captar los electrones que libera la planta al hacer su rizo deposición y así obtener la energía eléctrica por medio de una pila eléctrica, que transforma la energía química en energía eléctrica, es por medio de un electrodo positivo y un electrodo negativo, que atraen los electrones y protones lo que permite un flujo continuo debido a las reacciones que llevan a cabo en cada una de las etapas de la electrodeposición eléctrica.

La medición obtenida del contacto directo de los electrodos de Zinc y Cobre con la planta fue de 0.909 Volts, tanto para la Fase Luminosa como de la Fase Fijadora de Carbono, con una variación de \pm 0.05 volts, de acuerdo a las condiciones de humedad de la planta.

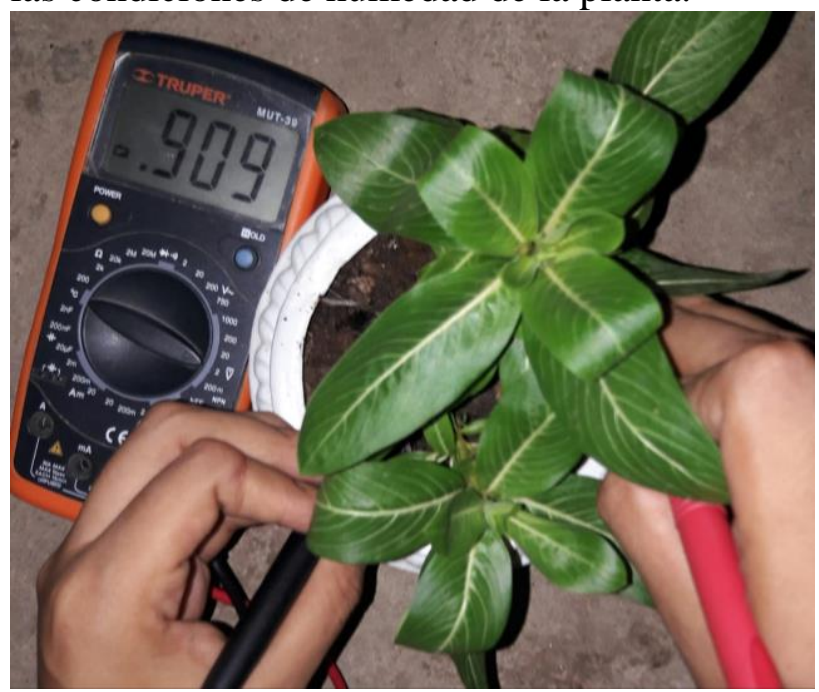

Figura 4 Medición Eléctrica en la Planta Fuente: Elaboración Propia

Los electrodos tenían una dimensión de $10 \mathrm{~cm}$ de largo con un grosor no mayor a los 0.5 $\mathrm{cm}$ de diámetro, por lo que considerando al ampliar el área para realizar la reacción redox sería mayor el flujo eléctrico se diseñó una malla de cobre para poder atrapar más electrones y obtener más voltaje mayor, dando como resultado una medición de 1.150 Volts presentando una mejora significativa del $26.5 \%$ en comparación con los electrodos convencionales de forma cilíndrica. 


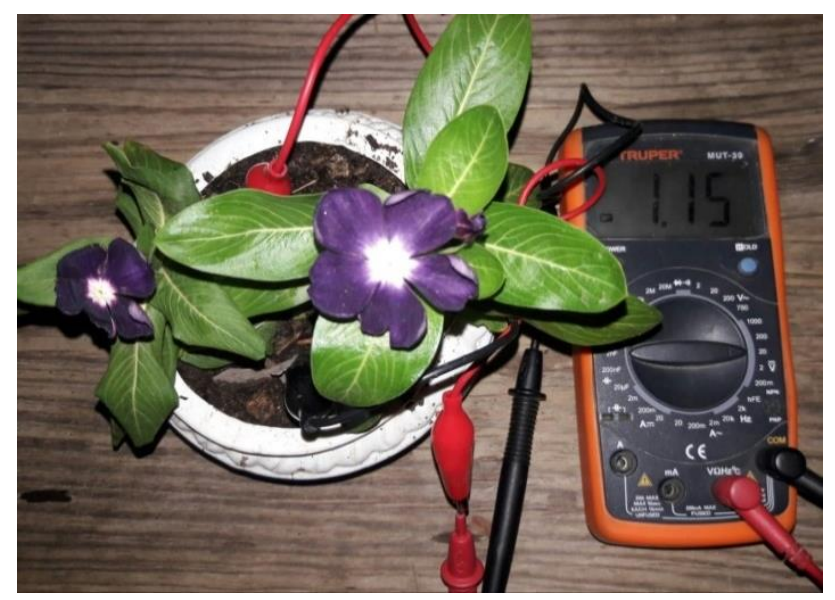

Figura 5 Medición Eléctrica con Diseño de Mallas Fuente: Elaboración Propia

Al considerar el sistema completo (planta - electrodos) como una celda de voltaje orgánica, se pueden realizar topologías mixtas (serie y paralelo) que permita aumentar el voltaje y corriente eléctrica para alimentar una carga física, también es factible combinar las celdas de voltaje (plantas) de diferentes familias. Una conexión en serie permite aumentar el diferencial de voltaje entre el electrodo inicial y final, replicando el diseño de mallas entre cada una de las plantas de prueba, se conectaron bajo esta topología los electrodos, lo que permitió alcanzar un voltaje de 3.872 Volts, suficiente para alimentar un par de leds como cargas en el circuito eléctrico.

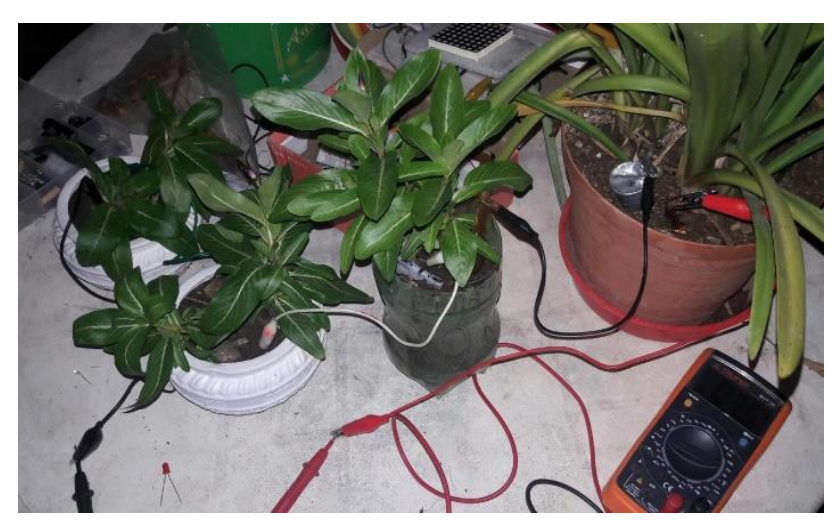

Figura 6 Topología en Serie de las Plantas Fuente: Elaboración Propia

\section{Conclusión}

Este proyecto permite identificar nuevas formas de generación eléctricas por medio de energías alternativas, renovables, sustentables y sostenibles, que no requiere una inversión de gran magnitud.
Con grandes ventajas ambientales al replicar y escalar esta propuesta a niveles de potencia que permitan alimentar cargas con valores nominales de 12 o 24 Volts en Corriente Directa, con la finalidad de posteriormente hacer uso de inversores y pasar a cargas de Corriente Altera a 120 Volts.

La investigación de esta tecnología aún tiene muchas vertientes, una de ellas es el uso de microorganismos que aumenten el proceso de descomposición orgánica y con ella del flujo de electrones a través de los electrodos, la experimentación realizada para la redacción de este artículo no cuenta con microorganismos añadidos a los que tiene el ecosistema per se para el desarrollo de la planta, lo que lleva concluir que las celtas de voltaje bajo una conexión en serie pueden aumentar su desempeño como lo han constatado diversas investigaciones previamente realizadas una vez que se encuentren dopadas de dichos microorganismos.

Otra línea de investigación que se pretende realizar al continuar con el proyecto es, fijar la atención a los valores de Corriente Eléctrica, hasta el momento solo se toma como referencia eléctrica el Voltaje (Diferencia de potencial Eléctrico), sin embargo para identificar la Potencia (Joules o Watts) y por ende la Energía obtenida del sistema de generación eléctrica propuesto, de esta manera permitirá comparar y de ser posible incorporar esta tecnología a los sistemas de generación distribuida tan ampliamente implementados alrededor del mundo.

De manera similar a la generación eléctrica por medio de los parques fotovoltaicos, donde el voltaje por cada celda individual es de apenas 0.7 Volts, la topología de conexión permite valores nominales de 12/24 Volts con corrientes de hasta 10 Ampers en los paneles solares, así mismo, la conexión serie-paralelo de las celdas orgánicas aumentara la potencia eléctrica generada.

El proyecto continua bajo investigación donde el siguiente paso a realizar es utilizar un metro cuadrado de césped natural con la base seccionada por medio de los electrodos que permita maximizar la energía eléctrica por área verde, estando consiente de la importancia de los espacios ecológicos y sus cuidados, mas ahora si es posible verlas como centrales eléctricas. 


\section{Referencias}

Antonio, C. S., David, B. D., Eduardo, C. F., \& Alonso, C. G. M. (2015). Generación distribuida, autoconsumo y redes inteligentes. Editorial UNED.

AQUINO-ROBLES, J., FERNÁNDEZ-NAVA, C., \& TRUJILLO-CABALLERO, J. (2016). La Enertrónica elemento clave en la transición hacia las redes eléctricas inteligentes en México. Revista de Investigación y Desarrollo, 2(6), 20-43.

Armaroli, N., \& Balzani, V. (2016). Solar electricity and solar fuels: status and perspectives in the context of the energy transition. Chemistry- $A$ European Journal, 22(1), 32-57.

Bacenetti, J., \& Fiala, M. (2015). CARBON FOOTPRINT OF ELECTRICITY FROM ANAEROBIC DIGESTION PLANTS IN ITALY. Environmental Engineering \& Management Journal (EEMJ), 14(7).

Cabrera, V., \& Foligno, L. (2001). Espontaneidad de las Reacciones Redox. In Anales de la Real Sociedad Española de Química (No. 1, pp. 23-25). Real Sociedad Española de Química.

Chen, H., Cong, T. N., Yang, W., Tan, C., Li, Y., \& Ding, Y. (2009). Progress in electrical energy storage system: A critical review. Progress in natural science, 19(3), 291-312.

Colcha, J. W., Cárdenas, M. J., Moncayo, C. R., Sisalima, J. D. Z., Heras, J. A. G., Solis, A. G. P., \& Yugsi, E. J. U. (2018). GENERADOR ENERGÉTICO A BASE DE ABONO INORGÁNICO Y ELECTROLITOS. FIMAQ Investigación y Docencia, 2(1).

Flexer, V., \& Mano, N. (2010). From dynamic measurements of photosynthesis in a living plant to sunlight transformation into electricity. Analytical chemistry, 82(4), 14441449.

González, N., Cusgüen, C., Mojica-Nava, E., \& Pavas, A. (2017). Estrategias de control de calidad de energía en microrredes rurales. Revista UIS Ingenierías, 16(2), 93-104. Lehmann, J. (2007). Bio- energy in the black. Frontiers in Ecology and the Environment, 5(7), 381-387.
Nguyen, K., \& Bruce, B. D. (2014). Growing green electricity: progress and strategies for use of photosystem I for sustainable photovoltaic energy conversion. Biochimica et Biophysica Acta (BBA)-Bioenergetics, 1837(9), 1553-1566. Reyes-Caballero, F., Fernández-Morales, F. H., \& Duarte, J. E. (2016). Panorama energético. Revista de investigación, desarrollo e innovación, 7(1), 151-163.

Roldán, J., \& Viloria, J. R. (2008). Fuentes de energía. Editorial Paraninfo.

Strik, D. P., Timmers, R. A., Helder, M., Steinbusch, K. J., Hamelers, H. V., \& Buisman, C. J. (2011). Microbial solar cells: applying photosynthetic and electrochemically active organisms. Trends in biotechnology, 29(1), 4149.

Wróbel, D. (2016). From natural photosynthesis to molecular photovoltaics. Molecular Crystals and Liquid Crystals, 627(1), 4-22.

Ying, C. Y., \& Dayou, J. (2016). Modelling of the electricity generation from living plants. Jurnal Teknologi, 78(6).

Zamora Silva, E. M. (2017). Comparación de la energía eléctrica generada mediante la fotosíntesis de las especies Ipomea purpurea y Palma areca, San Martin de Porres, 2017.

ZAPIEN-RODRÍGUEZ, José Manuel, SOLORIO-DE JESÚS, Bianca Azucena, BALLESTEROS-PACHECO, Juan Carlos y NÚÑEZAYALA, Frida Libertad. Generación Eléctrica a Partir de la Fotosíntesis Natural; ¿Una Realidad Escalable? Revista de Energías Renovables 2019 